\title{
极化原子束磁偏转法浓缩 $\mathrm{Eu}$ 同位素*
}

\author{
梅刚华 钟 达谭永芳 朱熙文 \\ (中国科学院武汉物理研究所, 波谱与原子分子物理国家重点实验室, 武汉 430071)
}

\section{关键词 Eu 极化原子束 磁偏转 同位素浓缩}

极化原子束有许多重要应用, 如用来研究原子散射过程 ${ }^{[1]}$, 制备极化核 ${ }^{[2]}$, 研究表面特 性 $^{[3]}$, 检验宇称不守恒 ${ }^{[4]}$, 浓缩同位素 ${ }^{[5 \sim 8]}$ 等等. 但是, 现有的工作都局限于碱金属原子, 而 多电子原子极化束制备和应用研究尚未见报道. 我们在 $\mathrm{Li}$ 和 $\mathrm{Rb}$ 原子束极化和同位素浓 缩 ${ }^{[6 ~ 8]}$ 的基础上,最近用一台连续波染料激光抽运, 制备出部分极化的稀土 $\mathrm{Eu}$ 原子束; 利用 其磁偏转效应, 观察到 $\mathrm{Eu}$ 的两种同位素的浓缩效果.

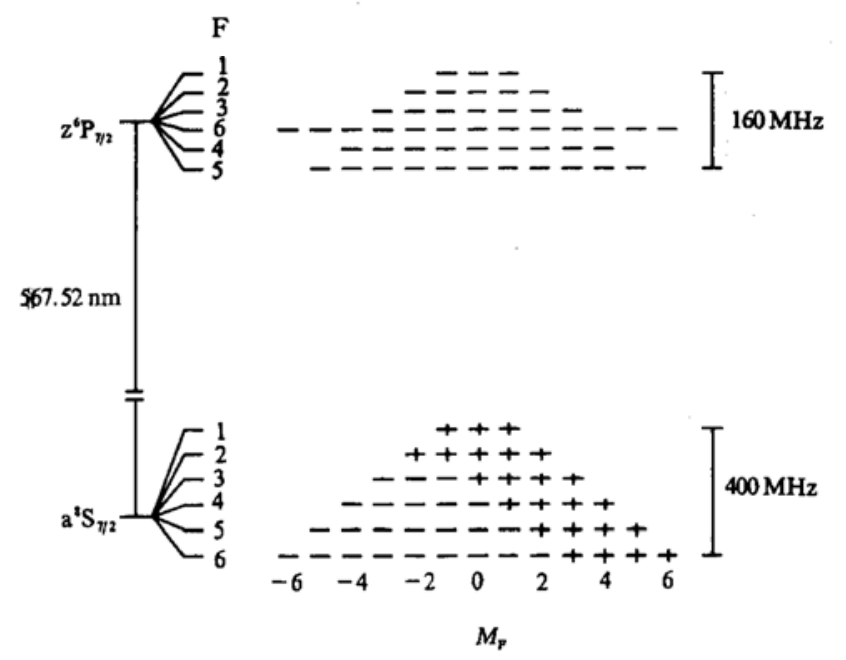

图 $1{ }^{151} \mathrm{Eu}$ 的 $\mathrm{a}^{8} \mathrm{~S}_{7 / 2}$ 和 $z^{6} \mathrm{P}_{7 / 2}$ 态超精细能级 (未按比例) $F$ 为总角动量量子数, $M_{F}$ 为磁量子数

天然 $\mathrm{Eu}$ 含核自旋 $I=5 / 2$, 丰度分别 为 $47.82 \%$ 和 $52.18 \%$ 的两种同位素 ${ }^{151} \mathrm{Eu}$ 和 ${ }^{153} \mathrm{Eu} .{ }^{151} \mathrm{Eu}$ 的基态 $\mathrm{a}^{8} \mathrm{~S}_{7 / 2}\left(4 \mathrm{f}^{7} 6 \mathrm{~s}^{2}\right.$ 组态) 和激发态 $z^{6} \mathrm{P}_{7 / 2}\left(4 \mathrm{f}^{7} 6 \mathrm{~s} 6 \mathrm{p}\right.$ 组态) 的超精细 能级示于图 $1\left({ }^{153} \mathrm{Eu}\right.$ 的 $\mathrm{a}^{8} \mathrm{~S}_{7 / 2}$ 的各 $\mathrm{F}$ 能级 间距, 以及 $z^{6} \mathrm{P}_{7 / 2}$ 的各 $\mathrm{F}$ 能级间距和顺序 与 ${ }^{151} \mathrm{Eu}$ 有所差别, 且二者的 $\mathrm{a}^{8} \mathrm{~S}_{7 / 2} \rightarrow$ $\mathrm{z}^{6} \mathrm{P}_{7 / 2}$ 谱线频率有约 $3.3 \mathrm{GHz}$ 的同位素移 位 $\left.{ }^{[9]}\right)$, 其中基态中的“+”和“ - ”分别表 示强磁场中具有正 $\left(m_{\mathrm{J}}>0\right)$ 、负 $\left(m_{\mathrm{J}}<0\right)$ 极化的超精细子能级. 由图 1 可知, 如用 光抽运方法实现高度的正、负极化, 即实 现全部负极化子能级粒子数向正极化子 能级的转移, 或全部正极化子能级粒子数 向负极化子能级的转移, 原则上须分别用 4 台激光同时抽运基态 $F=3,4,5,6$ 能级和 6 台激 光同时抽运全部 6 个基态 $F$ 能级. 而且, 抽运光还须有合适的偏振状态. 基态 $F$ 能级数目过 多 (碱金属只有两个), 是制备多电子原子极化束的主要困难. 但是我们发现, 有一些谱线几乎 重合 (如 ${ }^{151} \mathrm{Eu}$ 的 1 2(即基态 $F=1$ 到激发态 $F=2$ 跃迁谱线), 3 4 和 2 3 三条谱线间隔只 有 $20 \mathrm{MHz}$ 左右), 这就使得一台激光可以同时抽运几个 $F$ 能级, 从而使所需激光数目减少. 本实验中, 我们用一台激光器抽运, 仍然得到了明显的极化结果.

1995-10-05 收稿, 1996-01-08 收修改稿

* 国家自然科学基金和波谱与原子分子物理国家重点实验室资助项目 
实验装置与文献 [6]基本相同. 天然 $\mathrm{Eu}$ 原子从温度约为 $1000 \mathrm{~K}$ 的原子炉口溢出, 经准 直成束后在抽运区与垂直入射的 $\sigma^{+}$或 $\sigma$ 偏振激光束相交而被抽运极化. 激光光源为 Coherent 899-21 连续波激光器. 抽运区光强约为 $250 \mathrm{~mW} / \mathrm{cm}^{2}$, 激光束在原子束方向宽度为 $8 \mathrm{~mm}$, 频率在 $\mathrm{Eu}$ 的 $\mathrm{a}^{8} \mathrm{~S}_{7 / 2} \rightarrow \mathrm{z}^{6} \mathrm{P}_{7 / 2}$ 谱线 (576.52 nm) 附近扫描. 抽运区周围有一直流线圈, 提供强度 约为 $2 \times 10^{-4} \mathrm{~T}$, 方向与激光束平行、与原子束垂直的定向磁场, 以保证合适的激光偏振. 激光 的圆偏振度约为 $95 \%$. 抽运后原子进入六极磁铁的极孔. 六极磁铁磁极顶端的磁场约为 0.65 $\mathrm{T}$, 极孔直径为 $2.25 \mathrm{~mm}$, 长度为 $87 \mathrm{~mm}$. 六极磁铁的作用是将正极化原子聚焦, 负极化原子 发散. 因此, 如果穿过六极磁铁后某种同位素原子束强增大, 就说明该种同位素原子被正极化 并被浓缩; 反之, 如果减小, 则表明该种同位素原子被负极化, 而另一种同位素原子被浓缩. 原 子束强的变化用 Pt 热丝探测器探测. 在抽运区上方置有一光电倍增管, 探测光抽运过程中的 激光诱导荧光信号 (LIF), 与用热丝得到的原子束强信号同步记录. 将 LIF 谱与热丝信号比 较, 可以知道哪些谱线的共振抽运可导致 Eu 原子的极化. 4 种抽运方式下得到的束强变化和 LIF 信号示于图 2. 与 4 种抽运方式对应的激光波长和偏振分别为: (a) ${ }^{153} \mathrm{Eu}$ 的 $a^{8} \mathrm{~S}_{7 / 2} \rightarrow z^{6} \mathrm{P}_{7 / 2}$ 线, $\sigma^{+} ;(\mathrm{b})^{153} \mathrm{Eu}$ 的 $\mathrm{a}^{8} \mathrm{~S}_{7 / 2} \rightarrow \mathrm{z}^{6} \mathrm{P}_{7 / 2}$ 线, $\sigma^{-} ;$(c) ${ }^{151} \mathrm{Eu}$ 的 $\mathrm{a}^{8} \mathrm{~S}_{7 / 2} \rightarrow \mathrm{z}^{6} \mathrm{P}_{7 / 2}$ 线, $\sigma^{+} ;(\mathrm{d})^{151} \mathrm{Eu}$ 的 $\mathrm{a}^{8} \mathrm{~S}_{7 / 2} \rightarrow \mathrm{z}^{6} \mathrm{P}_{7 / 2}$ 线, $\sigma^{-}$. (a) 和 (c) 抽运使束强增大, ${ }^{153} \mathrm{Eu}$ 和 ${ }^{151} \mathrm{Eu}$ 同位素分别被正极化; (b) 和 (d) 抽运使束强 减小, ${ }^{153} \mathrm{Eu}$ 和 ${ }^{151} \mathrm{Eu}$ 同位素分别被负极化. 束强相对变化不大, 说明单一激光抽运只能实现 正、负极化子能级间的部分粒子数转移, 即部分极化. 图 2 中极化信号的峰高 $\Delta I$ 是一个正比 于正、负极化态粒子数差 $\left(N^{+}-N^{-}\right)$的物理量. 设磁铁对所有正极化子能级 (或所有负极化 子能级) 上的原子都具有相同的偏转特性, 即磁铁对 $m_{\mathrm{J}}$ 没有分辨 (文献 [10] 已证明这是一种 很好的近似), 可以推导出, 对于某种同位素 $\mathrm{A}$, 有 ${ }^{[11]}$

$$
P_{\mathrm{A}}=\left(N_{\mathrm{A}}^{+}-N_{\mathrm{A}}^{-}\right) /\left(N_{\mathrm{A}}^{+}+N_{\mathrm{A}}^{-}\right)=\Delta I_{\mathrm{A}} / I_{0} \beta_{\mathrm{A} 0} S,
$$

上式中 $I_{0} \beta_{\mathrm{A} 0}$ 表示没有光抽运时的总束强 $I_{0}$ 中天然丰度为 $\beta_{\mathrm{A} 0}$ 的同位素 A 所占的份额; $S$ 为

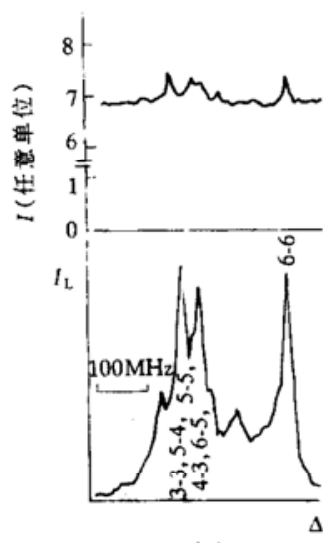

(a)

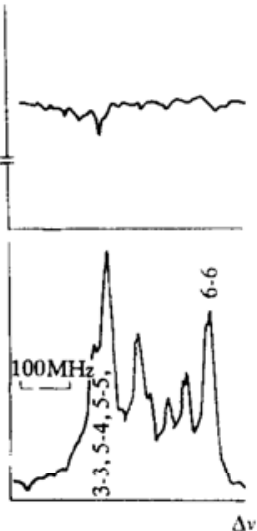

(b)

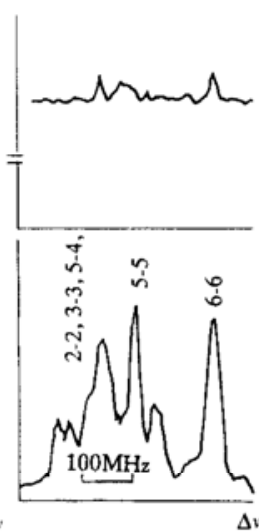

(c)

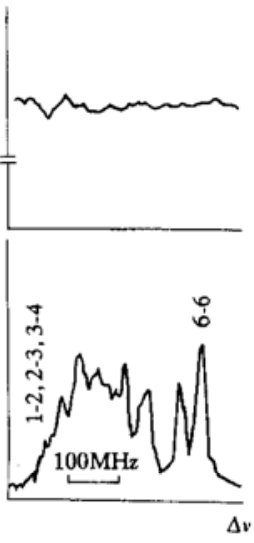

(d)

图 24 种抽运方式下得到的原子束强 $(I)$ 和 $\operatorname{LIF}$ 谱 $\left(I_{1}\right)$ $\Delta v$ 为扫描频率. 为明了起见, L.IF 谱仅标识了对应较大束强变化的共振谱线, 谱线标识是通过与文献 [9]的 Eu 原子高分辨 LIF 谱比较得到的 
磁铁的选择性, 定义为 $S=\left(\varepsilon^{+}-\varepsilon^{-}\right) /\left(\varepsilon^{+}+\varepsilon^{-}\right)$, 其中 $\varepsilon^{+}$和 $\varepsilon^{-}$分别表示正、负极化子能级上 原子穿过六极磁铁的几率. 根据(1)式可以对图 2 的结果进行半定量分析. 以图 2中 6-6 的 $\sigma^{+}$光抽运为例, 在抽运光为严格的右旋圆偏振, 且抽运光足够强, 抽运时间足够长的极限条件 下, 可由文献[11]所述方法, 根据跃迁几率计算出 $P=0.27$. 从图 2(a) 和 (c) 可得 $\Delta I / I_{0}$ 约为 $1 / 11, \beta_{\mathrm{A} 0}(A=151$ 或 153$) \approx 50 \%, S$ 是约为 0.9 的常数 ${ }^{[11]}$. 由此得到 $P$ 的实验值约为 0.2 . 考虑到实验条件与极限情况之间必然存在的偏差, 所以以上结果还是合理的. 由图 2 可看到, 在几个谱线交叠的地方都出现了极化信号 (理论分析表明, 若这些共振谱线完全不交叠, 这些 谱线的单独抽运只能产生较小甚至小到难以观察的极化信号), 此结果说明, 由于谱线交叠的 存在, 单一激光可以同时抽运数个基态 $F$ 能级, 从而使制备高度极化的 $\mathrm{Eu}$ 原子束所需的抽运 光源减少. 此结果对光抽运方法制备高度极化 $\mathrm{Eu}$ 原子束实验安排是有参考价值的. 原子极 化度与 $m_{\mathrm{J}}$ 有关, 热丝探测器对 $m_{\mathrm{J}}$ 没有分辨, 故由图 2 的结果不能确定极化度量值.

由图 2 可以定量确定同位素丰度的变化. (a) 和 (d) 对应 ${ }^{153} \mathrm{Eu}$ 的浓缩, (b) 和 (c) 对应 ${ }^{151} \mathrm{Eu}$ 的浓缩. 作为一般情况, 设光抽运使得穿过磁铁的同位素 $\mathrm{A}$ 的原子束强变化 $\Delta I_{\mathrm{A}}$ 同位素 $\mathrm{B}$ 的 原子束强变化 $\Delta I_{\mathrm{B}}$, 则可推导出同位素 $\mathrm{A}$ 的丰度为

$$
\beta_{\mathrm{A}}=I_{\mathrm{A}} /\left(I_{\mathrm{A}}+I_{\mathrm{B}}\right)=\left(\beta_{\mathrm{A} 0}+\Delta I_{\mathrm{A}} / I_{0}\right) /\left(1+\Delta I_{\mathrm{A}} / I_{0}+\Delta I_{\mathrm{B}} / I_{0}\right),
$$

推导中用到 $I_{\mathrm{A}}=I_{\mathrm{A} 0}+\Delta I_{\mathrm{A}}, I_{0}=I_{\mathrm{A} 0}+I_{\mathrm{B} 0}, \beta_{\mathrm{A} 0}=I_{\mathrm{A} 0} / I_{0}$, 在(2) 式中令 $\Delta I_{\mathrm{B}}=0$, 即对应于本实 验用单台激光抽运浓缩的情况. 另外, 显然可以由图 2 及(2)式推导出当有两台激光抽运时所 能达到的同位素丰度. 两台激光抽运的组合方式有两种: 1. (a) + (d), 将两台激光频率分别 调节到 (a), (d) 抽运方式的 $\Delta I_{153}$ 和 $-\Delta I_{151}$ 的极大处, 以浓缩 ${ }^{153} \mathrm{Eu} ; 2$. (b) + (c), 将两台激光频 率分别调节到 (b), (c) 抽运方式的 $-\Delta I_{153}$ 和 $\Delta I_{151}$ 的极大处, 以浓缩 ${ }^{151} \mathrm{Eu}$. 单台和两台激光抽 运下同位素丰度列于表 1 , 在得到表 1 的结果时. $\Delta I$ 均取束强变化的极大值. 极化信号的信噪 比约为 5 , 由此推算出表 1 中所列同位素丰度的实验误差约为 $1 \%$.

表 $1 \mathrm{Eu}$ 同位素浓缩效果

\begin{tabular}{|c|c|c|c|c|c|c|}
\hline \multirow{2}{*}{ 抽运方式 } & \multicolumn{4}{|c|}{ 单台激光抽运 } & \multicolumn{2}{|c|}{ 两台激光抽运 } \\
\hline & (a) & (b) & (c) & (d) & $(a)+(d)$ & $(b)+(c)$ \\
\hline$\beta_{153} / \%$ & 55.5 & 47.6 & 48.0 & 56.3 & 58.7 & 43.4 \\
\hline$\beta_{151} / \%$ & 44.5 & 52.4 & 52.0 & 43.7 & 41.3 & 56.6 \\
\hline
\end{tabular}

本文的结论如下:

(1)通过单台激光抽运, 得到了部分极化的稀土 $\mathrm{Eu}$ 原子束.

(2) 用极化原子束磁偏转方法, 观察到单台激光抽运时 ${ }^{153} \mathrm{Eu}$ 和 ${ }^{151} \mathrm{Eu}$ 的浓缩效果, 两种同 位素丰度改变在 $3 \% \sim 4 \%$ 之间; 由单台激光抽运浓缩结果, 推断出两台激光同时抽运所能达 到的 ${ }^{153} \mathrm{Eu}$ 和 ${ }^{151} \mathrm{Eu}$ 的浓缩效果, 两种同位素丰度变化约为 $7 \%$.

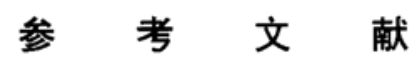

1 Blum K, Kleinpoppen $\mathrm{H}$. Analysis of scattering and excitation amplitude in polarized-electron-atom collisions. Phys Rev, 
1974, A9:1902

2 Calaprice F P, Happer W, Schreiber D F et al. Nuclear alignment and magnetic moment of ${ }^{133} \mathrm{Xe}^{133} \mathrm{Xe}^{\mathrm{m}}$ and ${ }^{131} \mathrm{Xe}^{\mathrm{m}}$ by spin exchange with optically pumped ${ }^{87} \mathrm{Rb}$. Phys Rev Lett, 1985, 54:174

3 Fick D. Surface physics with nuclear probes. Appl Phys, 1989, A49:343

4 Gilbert S L, Noecker M C, Watts R N et al. Measurement of parity nonconservation in atomic cesium. Phys Rev Lett, $1985,55: 2680$

5 朱然文. 通过极化原子束的磁偏转实现激光同位素浓缩. 物理学报, 1984, 33:1605

6 Zhu X, Huang G, Mei G et al. Laser isotope enrichment of lithium by magnetic deflection of a polarized atomic beam. J Phys, 1992, B25:3 307

7 黄贵龙, 梅刚华,朱熙文等. 半导体激光钢同位素浓缩实验。科学通报, 1992, 37(24):2 232

8 Mei G, Zhang Y, Huang G et al. Diode-laser isotope enrichment of rubidium with a polarized atomic beam. Appl Phys Lett, 1993, 63:3 568

9 Eliel E R, van Leeuwen K A H, Hogervorst W. Hyperfine anomaly in the $z^{6} \mathrm{P}_{7 / 2}$ level of the $4 \mathrm{f}^{7} 6 \mathrm{~s} 6 \mathrm{p}$ configuration of europium I. Phys Rev, 1980, A $22: 1491$

10 张 原, 朱熙文, 梅刚华. Eu 原子通过选态磁铁时的偏转特性. 物理学报, 1995, 44:685

11 Mei Ganghua, Huang Guilong, Wang Jiamin et al. Production of polarized ${ }^{7} \mathrm{Li}$ atomic beam in strong magnetic fields. Acta Phys Sin (overseas edition), 1993, 2:508 